\title{
LE MARCHE DE TRADUCTION AU CANADA ET AU NIGERIA : UNE ENQUETE COMPARATIVE
}

\author{
A COMPARATIVE STUDY OF THE TRANSLATION INDUSTRY IN CANADA AND \\ NIGERIA
}

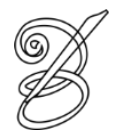

Olusegun AFOLABI

Doctorate candidate in Translation Studies

Université Laval (ULaval)

Québec City, Québec, Canada

olusegun.afolabi.1@ulaval.ca

Résumé : À l'ère actuelle qui est sans aucun doute marquée par la mondialisation, il n'est plus à démontrer que notre monde devient de plus en plus un village planétaire où tout se rapproche en rien de temps; et d'où la nécessite de faciliter la communication et surtout la compréhension entre les peuples et les pays. Partant de ce constat, on sera en ordre de rappeler que la traduction occupe donc une place importante dans la société moderne. Cependant, force est de constater que l'activité traductionnelle ne suit pas le même rythme d'un pays à l'autre et cela est dû à bon nombre de facteurs. Dans la présente étude, notre objectif premier est de démontrer les différents niveaux auxquels se situe le marché de traduction entre les deux pays qui constituent notre étude de cas, en l'occurrence le Canada et le Nigéria. Le but ultime de l'étude, qui se veut à la fois analytique, comparative, informative et correctionnelle, est de juxtaposer les deux contextes canadien et nigérian afin de voir comment l'un peut tirer profit de l'expérience de l'autre, pour ainsi contribuer au développement de la traduction dans le monde.

Mots-clés : Traduction. Marché de traduction. Traduction professionnelle. Le Nigéria. Le Canada.

Abstract: In the present age which obviously, is well known for globalization, the fact that the world is growing more and more into a global village where connectivity takes place in real time is no longer debatable. So also is the need to facilitate communication and understanding among people and countries. Based on this, it will not be wrong to recall that translation has thus become an integral part of the modern society. Meanwhile, it is equally important to note that due to certain factors, translation practices differ from one country to another. The purpose of this paper is, first and foremost, to show the differences that exist in the translation industry obtainable in Nigeria and Canada, the two countries that we have chosen as case study. The main aim of the study which is analytical, comparative, informative and corrective in approach is to compare and contrast the situation in the two countries under study, with a view to identifying how one can be enriched through the experience of the other, thus contributing to the development of translation in the world.

Key-words: Translation. Translation industry. Professional translation. Nigeria. Canada.

\section{Introduction}

$\int \begin{aligned} & \text { otre époque est celle de l'internet, de la mondialisation, de l'intégration } \\ & \text { nationale/régionale, des relations internationales, des médias sociaux, des nouvelles } \\ & \text { technologies, etc, mais aussi d'un grand besoin de la traduction. Comme on le }\end{aligned}$ réclame sans cesse, notre monde devient de plus en plus un village planétaire où tout se rapproche en rien de temps et d'où la nécessité de faciliter la communication et surtout la 
compréhension entre les peuples et entre les pays. On sera donc en ordre d'insister sur le fait que l'industrie de la traduction devient, d'ores et déjà, une partie intégrante de la société moderne car, comme l'a si bien souligné Cohen (2013), «... sans nous, c'est Babel! » En d'autres termes, si on comprenait Babel comme étant à l'origine de la multiplication des langues humaines, on comprendrait également que sans la traduction dans la communication inter linguistique, ce sera la confusion!

Face à ce développement intéressant, force est de savoir que le courant de l'activité traductionnelle ne se passe pas de la même manière sur tous les continents et dans tous les pays. Tandis que certains pays ont vite reconnu le pouvoir de traduire, d'autres ne l'ont pas encore fait. En d'autres termes, certains pays sont conscients de l'indispensabilité du traducteur plus que les autres et c'est en cela que se situe la problématique de la présente étude. Il s'agit de démontrer les différents niveaux où se situe le marché de traduction entre les deux pays qui constituent notre étude de cas, en l'occurrence le Canada et le Nigéria. Le but ultime de l'étude, qui se veut à la fois analytique, comparative, informative et correctionnelle, est de juxtaposer les deux contextes canadien et nigérian afin de voir 74 comment l'un peut apprendre de l'autre. On peut dire que le choix de comparer le marché de traduction au Canada et au Nigéria trouve sa justification dans le fait que la coopération canado-nigériane existante depuis fort longtemps (AIRE, 2014, p. 13) mérite d'être mise en exergue, encouragée, voire améliorée.

L'étude va donc commencer avec un aperçu historique de l'activité traductionnelle dans les deux pays ciblés et cela en vue d'apprécier le chemin parcouru jusque-là. Ensuite, on fera état de la situation actuelle dans les deux contextes en touchant aux aspects pointus comme les besoins et les opportunités dans le marché de traduction, l'organisation du marché, la formation de base requise pour intégrer le marché et la formation continue nécessaire pour s'y établir. Le reste de l'étude se concentrera sur les opérateurs du marché, les regroupements professionnels et le réseautage dans le marché, la tarification et la question du contrôle de la qualité. Finalement, on examinera les défis du marché et les perspectives d'avenir dans les deux contextes étudiés. Il faudra comprendre que dans le souci pour la précision et étant donnée la primauté du Québec dans le marché de la traduction au Canada, cette étude se basera plutôt sur le marché de traduction dans la province du Québec. On peut se demander pourquoi ce choix de Québec en particulier. C'est tout à fait parce que nous avons compris, par exemple grâce à Cloutier (2013, p. 9) que le Canada représente un peu moins de 0,5\% de 
la population mondiale et occupe $10 \%$ du marché mondial de la traduction; et surtout qu'environ $90 \%$ de cette traduction se fait dans et pour la belle province, le Québec.

\title{
2. Aperçu historique de la traduction au Canada et au Nigéria
}

À en croire Cohen (2013),

\begin{abstract}
S'il est un endroit où les traducteurs ont un marché établi, c'est bien le Canada. Et le Québec, au sein du Canada, jouit d'une situation encore plus privilégiée. Cet état de fait est dû à deux événements importants : la création, en 1934, d'un bureau central de la traduction devant servir tous les ministres du gouvernement fédéral et l'adoption, en 1976, de la charte de langue française par le gouvernement du Québec.
\end{abstract}

C'est l'image ci-dessus qui décrit exactement l'origine du marché de traduction au Canada et depuis lors, le marché ne cesse de grandir et de fleurir. Grâce à un bilinguisme officiel bien établi depuis plus de 40 ans, le Canada s'est taillé une place de choix sur le marché de la traduction (LYAN, 2014).

En de termes plus précis, l'histoire de la traduction au Canada ne date pas seulement d'hier, cela date de décennies. Donc, le marché de traduction y est un marché assez robuste, fortement nourri du bilinguisme officiel du pays. C'est aussi la raison pour laquelle sur le plan mondial, on reconnaît le Canada comme la terre de la traduction.

Quant au Nigéria, bien que l'histoire nous apprenne qu'en 1845, la Sainte Bible était traduite de l'anglais vers le yorouba - l'une des langues nationales du pays - par Samuel Ajayi Crowther, un ancien esclave des colons devenu évêque, le vrai marché de traduction remonte au $20^{\mathrm{e}}$ siècle. Selon Asobele (1999, p. 18), les colonisateurs se servaient des traducteurs communautaires pour faciliter la communication entre eux et les colonisés. Mais la traduction n'a pas eu de valeur commerciale en tant que profession dans le pays avant la fin de la colonisation.

Par ailleurs, il faudrait noter que les écrivains nigérians traduisaient les œuvres écrites en langues étrangères vers les langues nigérianes et vice versa. C'est ainsi que John Abioye, un ancien professeur de français à l'Université de Lagos, a pu traduire quelques œuvres de J.O Fagunwa de la langue yorouba vers le français. De la même manière, le lauréat nigérian du prix Nobel pour la littérature (1986), le professeur Wole Soyinka a traduit certains romans yoroubas en anglais. Beaucoup d'universitaires nigérians ont également, soit traduit les œuvres existantes en langues nationales nigérianes ou étrangères, soit fait la critique des 
œuvres traduites par les autres. Bref, il faut rappeler qu'au début, c'était la traduction littéraire qui était très pratiquée au Nigéria, et non la traduction en tant qu'une activité professionnelle.

Cependant, nous pouvons dire que c'était le 'Negro-African Festival of Arts and Culture (FESTAC)' tenu à Lagos entre janvier et février 1977 qui a rendu le marché de la traduction professionnelle très visible au Nigéria. Grâce à cet événement panafricain qui a rassemblé les noirs venus de tous les continents du monde, les besoins de la traduction se sont fait ressentir et c'est à partir de ce moment-là que le marché a réellement commencé à prendre de l'essor.

D'après Asobele (1999, p. 60) :

\begin{abstract}
During this cultural festival, the services of the small number of professionally trained African translators and interpreters were stretched to the limit, and Nigeria alone with its five existing Universities in Ibadan, Lagos, Zaria, Ile-Ife and Nsukka (at that time) could not train the bilingual linguists necessary to service the various sectors of the cultural festival [...] Little wonder then that Dr. Ekundayo Simpson who served as director of translation at the National Secretariat of FESTAC ' 77 and a Canadian trained translator came to grips with this endemic problem $[\ldots]$
\end{abstract}

76 En outre, l'implantation des entreprises multinationales d'origine française telles Peugeot, Total, CFAO, etc., et la création des organisations internationales comme la CÉDÉAO (Communauté économique des États de l'Afrique de l'Ouest) avec son siège d'abord à Lagos, puis à Abuja-la nouvelle capitale fédérale du Nigéria, entre autres, ont fait que la demande des services de traducteurs et interprètes augmentent et cela a également élargi le marché. Aujourd'hui, la profession de traduction est très populaire au Nigéria même si le marché n'est toujours pas bien coordonné comme nous allons le voir plus loin.

\title{
3. Besoins et opportunités du marché
}

Nul ne doutera du fait que dans le monde en général, les besoins de la traduction deviennent de plus en plus grands et les opportunités aussi ne cessent de grandir pour les traducteurs professionnels dignes du nom.

Au Canada, le marché de la traduction connaît une forte croissance. Cette croissance provient des besoins toujours plus grands en information. On la retrouve ainsi dans le secteur public, mais surtout dans le secteur privé. Les secteurs qui offrent les meilleures perspectives à cet égard sont : l'aérospatiale, les transports, les services aux entreprises, l'industrie pharmaceutique, les équipements de télécommunication, les services financiers, les technologies de l'information et les organisations internationales. En effet, selon Service 
Canada (2015), la mondialisation de l'économie implique que les entreprises doivent traduire leur documentation dans les langues des pays qui consommeront leurs produits ou services. Ainsi, en plus du volume traditionnel de traduction entre l'anglais et le français, la demande locale de traduction multilingue (espagnol, allemand, italien, etc) est en hausse. Les donneurs d'ouvrages préfèrent en effet faire affaire avec une seule maison de traduction pour l'ensemble de leurs besoins en traduction.

Non seulement la documentation accompagnant les produits doit être comprise dans plusieurs langues, mais, comme ces produits sont de plus en plus complexes, les guides les accompagnants sont beaucoup plus longs qu'auparavant. Pensons par exemple à la multiplication des fonctions offertes par les produits électroniques qui doivent être décrites en détail et clairement de façon à être comprises par les utilisateurs. Ce facteur génère lui aussi une croissance du volume de travail des traducteurs.

La croissance des besoins en information est encore accentuée par le développement des services offerts sur Internet ou sur des intranets d'entreprises. Que ce soit du côté des services gouvernementaux ou des services offerts par les entreprises privées, le développement de ces services génère un volume de travail très significatif pour les traducteurs, tant pour la création des sites que pour la mise à jour de leur contenu (SERVICE CANADA, 2015).

Même si pour le Canada c'est beaucoup plus significatif, l'image brossée ci-dessus va de même pour le Nigéria en termes des besoins et des opportunités du marché de traduction. D'abord, la complexité linguistique du Nigéria avec plus de 500 langues parlées explique, en partie, les besoins de traduction comme un moyen de faciliter l'entente et promouvoir l'intégration nationale. En plus, l'emplacement géographique du pays qui se voit entouré entièrement des pays officiellement francophones et le rôle prépondérant que joue le Nigéria dans la sous-région ouest-africaine, même dans le monde entier, y favorisent dans une grande mesure le marché de traduction.

Ce n'est donc pas étonnant que de nombreuses rencontres internationales faisant appel au service de traducteurs ponctuent l'année au Nigéria. Les entreprises multinationales, les activités d'importation et d'exportation sont, elles aussi, des opportunités qui font fleurir le marché de traduction au Nigéria. 


\section{Organisation ou structure des marchés canadien et nigérian de traduction}

Comme nous l'avons mentionné ci-dessus, le Canada est un pays où la profession de traduction est très bien organisée et reconnue, comparativement à ce que l'on constate au Nigéria. Tandis que le gouvernement fédéral canadien dispose d'une politique bien définie qui reconnait l'importance de la profession à tel point qu'il existe un bureau gouvernemental fédéral de traduction, le gouvernement nigérian n'a pas encore accordé une telle importance à la profession, en dépit du grand besoin de la traduction dans ce pays très multilingue.

Selon le Service Canada (2015), environ $53 \%$ des traducteurs, terminologues et interprètes travaillent dans les services de traduction et d'interprétation, et $11 \%$ dans l'administration fédérale. Les autres se répartissaient dans un grand nombre d'industries, avec une certaine concentration dans les secteurs de la finance et des assurances (4\%) de l'information et de la culture $(4 \%)$, et de l'enseignement $(4 \%)$. D'après les dernières estimations, le Canada détiendrait en effet $10 \%$ du marché mondial de la traduction professionnelle, estimé à 31 milliards de dollars en 2012, et serait sur le point d'atteindre les 33 G\$. Près de $49 \%$ de ces revenus proviendraient du marché québécois. Une forte présence 78 qui se ressent également sur les effectifs, puisque, des 25000 entreprises de services langagiers présentes au niveau mondial, le Canada emploie près de 15000 traducteurs, interprètes et localisateurs pour un chiffre d'affaires de $3 \mathrm{G}$.

Au Canada, et particulièrement au Québec, les traducteurs travaillent pour des cabinets de traduction ou pour les gouvernements comme salariés ou à leur compte, comme travailleurs autonomes (COHEN, 2006, p. 13). Le travail à temps partiel est fréquent. En outre, plusieurs traducteurs occupent plus d'un emploi, comme rédacteur par exemple, et à l'inverse, plusieurs membres d'autres professions font de la traduction, souvent dans des domaines bien précis : acteurs, programmeurs, rédacteurs, enseignants, etc. Les emplois sont proportionnellement plus présents dans l'Île de Montréal et dans l'Outaouais que dans les autres régions (SERVICE CANADA).

Dans le cas du Nigéria, il est présentement difficile de chiffrer exactement combien de traducteurs sont en train d'exercer sur le territoire national car aucune étude n'a été menée à cet effet. Aussi, comme cela a été mentionné plus haut, le marché de traduction au Nigéria est tellement vaste, ouvert et sans contrôle quelconque, d'autant plus que n'importe qui peut se présenter comme traducteur, une fois que la personne comprend l'anglais et le français ou d'autre langues demandées, ce qui mine sérieusement la bonne pratique de la profession. Il est clair que la plus grande partie de ceux qui se ventent comme traducteurs au Nigéria 
aujourd'hui sont des licenciés en langue française qui n'ont eu aucune formation professionnelle en traduction. La réalité est que présentement, au Nigeria, le nombre de traducteurs qualifiés est hautement inferieur au volume de services de traduction disponible (SIMPSON, $2007: 258$ ).

Néanmoins, il convient de noter qu'il existe quelques cabinets de traducteurs exerçant présentement sur le marché nigérian, notamment, Interlingua Limited géré par le Professeur Ekundayo Simpson-le doyen de la traduction au Nigéria (ASOBELE, 1999 ; JOHNSON, 2004), Multitongue and Ears Limited, Translat Ltd, parmi tant d'autres. Mais, il faut retenir que beaucoup d'entre ces cabinets n'ont pas de traducteurs salariés, contrairement à ce qui s'obtient au Canada. La pratique de travail à la pige est la plus populaire dans le marché de traduction au Nigéria. C'est-à-dire, le traducteur est engagé lorsqu'il y a du travail à donner par son cabinet de rattachement et il est payé en fonction de ce qu'il fait. Il n'y a vraiment pas d'emploi permanent dans ce cas. C'est la raison pour laquelle, à l'exemption de ceux qui sont chanceux d'être engagés par des organisations internationales comme la CÉDÉAO, l'UA, l'UNESCO, etc., la plus grande partie des traducteurs exerçant au Nigéria sont des «freelancers », c'est-à-dire les travailleurs autonomes ou les pigistes.

En outre, il n'est pas rare de trouver ceux qui abusent de l'usage des nouvelles technologies notamment celles «facilitant» la traduction automatique. Le plus souvent, au lieu de faire appel aux traducteurs professionnels, quelques bricoleurs-traducteurs se servent à tort et à travers des logiciels de traduction, croyant que c'est du passe-partout. Comme on peut déjà l'espérer, cette voie ne mène qu'à des fausses traductions surtout lorsqu'elle est empruntée par des novices ou même des traducteurs non avertis. C'est certainement ceci qui pousse SÉGARD (2009) à remarquer ce qui suit :

\footnotetext{
Je pense personnellement que la technologie joue un rôle important dans le domaine de la traduction en général. J'entends bien sûr un certain type de technologie. Les outils de «traduction » que l'on trouve dans une section spéciale de moteurs de recherche tels que Google (Outils linguistiques), Yahoo (Babel Fish), Reverso, SYSTRANet, etc. sont quant à eux bien loin de remplacer la traduction humaine. Ces outils sont très limités en ce sens qu'ils négligent de nombreux aspects du texte source et une grande quantité de facteurs qu'un cerveau humain normalement constitué et formé à la traduction professionnelle peut traiter et prendre en compte dans le processus complexe de la traduction, à savoir le style, le domaine de spécialisation, la terminologie, les différences culturelles, le contexte, etc. (C'est nous qui soulignons)
}

Toujours au sujet de l'organisation du marché de traduction, il est intéressant d'apprendre que les groupements professionnels de traducteurs fonctionnent très bien au 
Canada. Par exemple, au Québec, selon ce que nous lisons du Service Canada, l'Ordre des traducteurs, terminologues et interprètes agréés du Québec (OTTIAQ) compte à lui seul près de 2000 membres et il est très actif dans le marché.

Bien que l'appartenance à l'ordre ne soit pas obligatoire pour exercer la profession de traduction au Québec, certaines instances gouvernementales et cabinets de traductions n'engagent que les traducteurs agréés par l'OTTIAQ. Par exemple, durant notre recherche d'emploi comme traducteur/interprète, certaines entreprises que nous avons contactées ont demandé si nous étions membre de l'ordre professionnel et en répondant par 'non, pas encore', ils ont été peu enthousiastes de nous engager car ils sont d'avis que l'appartenance à l'ordre professionnel est un atout. Ceci souligne l'importance de l'OTTIAQ dans le marché de traduction au Québec.

Par contre, au Nigéria, nous pouvons dire que le marché de traduction est ouvert à n'importe qui le veut. À présent, il existe un rassemblement professionnel de traducteurs appelé « Nigeria Institute of Translators and Interpreters (NITI) » mais la vérité est que NITI ne peut pas se mettre au même pied d'égalité que l'OTTIAQ sur le plan de l'engagement

80 professionnel. En fait, contrairement au Canada où l'OTTIAQ et les autres regroupements professionnels de traducteurs dans les autres provinces jouissent d'une reconnaissance officielle du gouvernement, le NITI du Nigéria n'est pas béni de la même sorte. Aucune reconnaissance constitutionnelle pour le moment car le projet de loi du NITI attend toujours d'être voté par l'Assemblée nationale du Nigéria et cela depuis plus de dix ans, en dépit des efforts inlassables des pionniers de l'industrie, notamment le Professeur Ekundayo Simpson.

\section{Comment intégrer le marché (Quelle formation de base requise?)}

Pour intégrer le marché de traduction au Canada, il est bel et bien clair que ce n'est pas donné à n'importe qui d'y arriver. Le gouvernement du Canada a investi dans la création de programmes de traduction qui sont disponibles dans bon nombre d'universités canadiennes, notamment l'Université Laval, l'Université de Montréal, l'Université McGill, l'Université d'Ottawa, pour ne citer que celles-là (VALENTINE, GUEVEL, 1997).

Selon le Service Canada,

Pour accéder à cette profession, il faut habituellement être titulaire d'un baccalauréat en traduction ou dans une discipline connexe et une spécialisation en traduction, en terminologie ou en interprétation. En outre, une formation dans un domaine spécialisé en demande (droit, génie, informatique, pharmacie, économique, etc.), jumelée à une très bonne connaissance de la langue de départ et à une parfaite 
maîtrise de celle d'arrivée, peut aussi permettre d'accéder à certains postes spécialisés. La connaissance approfondie d'une troisième langue représente un atout et est parfois exigée.

En plus de ce qui est présenté ci-dessus, le traducteur, le terminologue et l'interprète agréés pratiquent une profession à titre réservé. Ils doivent détenir un permis de l'Ordre des traducteurs, terminologues et interprètes agréés du Québec et être inscrits au Tableau de l'Ordre pour utiliser le titre réservé, soit « traducteur agréé », « terminologue agréé », « interprète agréé » ou le genre féminin, soit les abréviations «trad.a. », « term.a. », « int.a. » ou leurs versions anglaises respectivement. Pour obtenir son permis, le candidat doit détenir un diplôme québécois prévu par règlement ou encore un diplôme ou une formation reconnus équivalents par l'Ordre. Il n'est pas nécessaire d'être résident permanent ou citoyen canadien pour obtenir un permis. Cela veut dire que l'octroi du permis est ouvert à tous ceux qui remplissent les conditions d'admissibilité énumérées ci-dessus, peu importe leur statut de résident au Québec. De plus, chaque membre de l'OTTIAQ possède un sceau qu'il doit mettre sur les copies de son travail à titre d'attestation officielle ou professionnelle.

En ce qui concerne le Nigéria, puisqu'il s'agit, pour le moment, d'un marché qui n'est pas très organisé, il n'y a pas de condition stricte pour exercer la traduction, surtout en tant que «freelancer». Il n'y a que les organismes internationaux et quelques entreprises multinationales qui recrutent des traducteurs pour leurs services qui insistent sur l'obtention de diplômes professionnels comme condition requise pour être engagé. Contrairement à ce qui s'opère au Canada, le gouvernement nigérian n'investit pas encore dans la formation professionnelle des traducteurs, ce qui influe négativement sur le marché de traduction et son rendement en général. Cela laisse aussi le marché de traduction au Nigéria ouvert et donc, envahi par les traducteurs étrangers venant des pays francophones voisins. En effet, il mérite d'être répéter ici que le marché nigérian de traduction est si grand mais les traducteurs professionnels nigérians sont peu nombreux.

\section{Tarification}

Chez les traducteurs canadiens, le mode traditionnel de tarification est la tarification au mot. Généralement, le tarif varie entre 0,16 et 0,18 cents (de dollar canadien) le mot pour la traduction de l'anglais vers le français, et entre 0,20 et 0,21 cents le mot s'il s'agit de traduire du français vers l'anglais. Évidemment, au Québec, le traducteur anglais gagne 
beaucoup plus que son collègue français et pour cause, la plupart des documents au Québec sont rédigés en français pour être traduits en anglais.

Un point important à retenir concernant la façon dont le traducteur détermine le nombre de mots dans son texte, selon DE VOS (2009), est celui-ci : « Avant l'arrivée des ordinateurs personnels, le compte de mots devait se faire «à la main », ce qui pouvait être assez exigeant pour un document volumineux. Mais maintenant qu'il existe des compteurs de mots intégrés aux logiciels de traitement de textes, l'exercice est devenu un jeu d'enfant. Toutefois, certains traducteurs préfèrent facturer à l'heure, pour diverses raisons...»

Cependant, il faut rappeler que depuis un certain moment, le débat sur le choix à privilégier entre la tarification au mot et à l'heure ne cesse de se présenter sur le marché et occupent les professionnels langagiers au Canada à tel point qu'un numéro de Circuit (le journal de l'OTTIAQ) a été consacré à cette thématique. Tandis que certains sont pour la tarification au mot, d'autres soutiennent très fortement la tarification à l'heure (voir par exemple LAVALÉE, GAUTHIER et BOHEN, 2009).

Au Nigéria, par contre, la tarification est souvent faite par nombre de pages et dans 82 quelques circonstances par nombre de mots aussi. Une page de 250 mots au maximum est facturée à 25 \$ (dollar américain) et il n’y a pas tellement de différence entre le taux de tarification des textes anglais-français ou vice versa. Certains contrats sont facturés par jour au Nigéria, par exemple, s’il s'agit d'une conférence. Dans ce cas, le traducteur est payé entre 200 \$ et 250 \$ par jour, selon le contrat.

Encore faut-il ajouter qu'en général, le taux officiel, si jamais il en existait un, n'est guère respecté à cause du manque de coordination du marché. Selon ce que AMOSUN (2010) nous laisse entendre, «The professional rates are regularly sabotaged as a result of ignorance on one hand, and on the other hand, what one must call 'profiteering' by those who require our services. »

\section{Assurance de la qualité et les défis du marché}

S'il y a une profession où l'assurance de la qualité doit être de mise, c'est bien celle de la traduction car une mauvaise traduction peut engendrer de très gros dégâts, voire la guerre (ASOBELE 1999: 16). Ayant compris cela, au Québec, l'OTTIAQ fait tout le nécessaire pour protéger le public qui est sa clientèle en assurant la qualité de la prestation de ses membres. Par conséquent, à part la formation continue qu'organise l'Ordre régulièrement à l'intention de ses membres, une équipe de l'Ordre entreprend des visites d'inspection 
professionnelle régulièrement auprès de ses membres. «Tous les membres agréés actifs inscrits au Tableau des membres de l'Ordre sont assujettis à l'inspection professionnelle en vertu du code des professions. Impossible d'y échapper », nous informe (MESA, 2013, p. 6). Paquette, citée par Mesa ajoute que cette inspection professionnelle n'est pas un moyen de coercition, mais c'est plutôt comme un outil de prévention et formation.

Malheureusement, ce genre de mesure de contrôle de qualité n'existe guère dans le marché de traduction au Nigéria. Comme cela a été mentionné plus haut, c'est un marché ouvert à tous (AMOSUN 2010, p. 78). «It's an all comers affair » comme on le dit en anglais nigérian. Il n'y a aucun contrôle tout simplement parce que le NITI du Nigéria qui devrait jouer le même rôle que l'OTTIAQ au Québec cherche toujours à s'établir du point de vue constitutionnel, d'où la difficulté, pour le moment, de pouvoir régir l'industrie de traduction comme il se doit. Pour pallier à ce problème dans le marché nigérian de traduction, voici ce que propose Amosun, le président en exercice du NITI :

\begin{abstract}
The future of translation and interpretation remains rosy in spite of the dangerous activities of interlopers who have no stake in the sector. I would warmly welcome the idea of recourse to personal, NITI registered stamps on EVERY translation job just as conference interpreters must be listed on official performance reports on interpretation teams. Such reports would then be stored for reference purposes. (2010, p. 79)
\end{abstract}

On espère que dans un proche avenir et avec la ratification tant escomptée du projet de loi en faveur de la reconnaissance officielle du statut du NITI par le gouvernement fédéral nigérian, le problème de l'assurance de qualité qui présentement est un grand défi du marché de traduction au Nigéria sera considérablement réduit, sinon complètement occulté.

Parallèlement, l'expérience personnelle de Ségard Virgine citée ci-dessous nous apprend de sérieuses leçons à l'égard de ceux ou celles qui opèrent le 'marché au noir' dans le marché de la traduction, qui est supposé être professionnel au sens stricte du mot. Elle atteste au fait que les défis de l'assurance de qualité et de la tarification sont presque partout semblables.

Le traducteur professionnel est malheureusement victime d'une espèce sans scrupules que j'appellerai le «bricoleur des mots », celui-là même qui ôte le pain de la bouche du professionnel en offrant des services de pacotille à des tarifs dérisoires. C'est ainsi que j'ai vu une offre me passer sous le nez en proposant un tarif de $20 £$ pour une page de traduction de 109 mots, ce qui me paraissait tout à fait raisonnable étant donné qu'un traducteur professionnel a conscience de ce qu'il vaut et propose toujours un tarif minimum. D'autant plus raisonnable qu'il s'agissait d'un message d'Erin Brokovitch à des travailleurs ayant été exposés à l'amiante et souffrant, trente 
ans plus tard, de mésothéliome et de cancer du poumon. Je ne sais pas ce que vous en pensez, mais personnellement, je ne plaisante pas avec la traduction et encore moins avec des textes de ce genre. Un autre hurluberlu s'est prostitué en acceptant de faire la traduction pour $5 £$, certainement pardessus la jambe, exactement comme une prostituée en fin de compte... Eh oui, traduire à de tels tarifs, c'est se prostituer. (SEGARD, 2009)

\section{Perspectives d'avenir : le plaisir de traduire ou la crainte de survivre?}

Certainement, l'expérience que nous venons de citer ci-dessus ne sera pas limitée à cette seule auteure, d'autres aussi en auraient eu la même (même pires) à un moment donné de leur parcours de carrière de professionnel langagier. Il reste donc un défi à relever pour que le plaisir de traduire ne cède pas la place aux craintes de survivre. Quoiqu'il en soit, toujours estil que ce qui est bon reste toujours bon et ce qui n'est pas bon n'est pas bon. Autrement dit, les donneurs d'ouvrages reconnaissent la qualité, et les bricoleurs ne feront pas long feu avant d'être reconnus dans le marché. Donc, pour le traducteur qui connaît bien son métier et qui respecte la déontologie de la profession, pas de crainte.

Cela étant, force est d'admettre que l'avenir du marché de traduction au Canada est prometteur et porteur d'espoir. À en croire Cohen (2013), «les perspectives sont, pour l'instant, favorables [...]»D'autres acteurs dans l'industrie voient également l'avenir du marché tout en rose, en dépit de quelques défis du présent.

De la même manière, dans le cas du Nigéria, l'avenir n'est pas trop menacé non plus, vu l'abondance de potentialités pour le développement du marché de traduction/interprétation dans le pays. Ce qui y reste à avoir, c'est la bonne volonté, tant de la part du gouvernement que de la part des opérateurs du marché, les traducteurs eux-mêmes. Tout ce que le Canada peut faire en matière de la profession de traduction, le Nigéria le peut aussi, nous le croyons.

À cet égard, nous voudrions rappeler cet avis encourageant du Service Canada selon lequel « la hausse de la demande en services de traduction est telle que les besoins de traducteurs devraient continuer à augmenter de façon notable au cours des prochaines années, malgré l'utilisation de la traduction automatique et la hausse de la productivité [...] » Ce constat, à notre avis, va de pair tant pour le Canada que pour le Nigéria, dans une perspective de l'avenir.

\section{Conclusion}

Selon un témoignage personnel d'Aire (2014, p. 13), 
toutes les proportions gardées, le Canada marqué, entre autres, par un bilinguisme très performant, demeure une terre d'accueil pour de nombreux compatriotes.

Eu égard à ce constat, il tombe dans le bon sens donc de s'intéresser davantage à l'industrie de la traduction comme étant un intérêt commun partagé par le Canada et le Nigéria, et de voir comment l'un peut tirer profit de l'autre. D'où la pertinence scientifique et sociale de la présente étude.

Sommairement, ce sont donc les résultats d'une brève enquête comparative menée sur le marché de traduction au Nigéria et au Québec, Canada que nous nous venons de présenter ici. Sans prétendre à une exhaustivité quelconque, nous sommes d'avis que les informations fournies dans cette étude pourront nourrir de plus profondes réflexions qui vont aider à maintenir le statut appréciable déjà établi pour le marché de traduction au Canada, et à améliorer le niveau du marché nigérian qui, selon toutes les apparences, doit encore progresser, mais qui est quand même plein de potentialités. Nous espérons également que les étrangers comme nous, désireux de s'intégrer sur le marché de traduction canadien trouveront dans ce travail, quelques pistes pouvant aider à mieux les orienter, pour ne pas être induits en erreurs en tant que nouveaux-venus ou des «first timers or Johnny-just-come », comme on le dit en pidgin nigérian.

Nous terminerons nos propos ici dans une perspective pédagogique en soulignant le fait qu'une solution particulière pour amener le niveau du marché de traduction au Nigéria prés de celui du Canada consistera à prendre la question de la formation professionnelle des traducteurs/interprètes beaucoup plus au sérieux. S'il n'est plus à débattre que la formation est l'épine dorsale de toute profession, c'est exactement ce que le Canada a su faire depuis très longtemps en créant de programmes de formation professionnelle des traducteurs dans ces universités. Et nous pouvons dire que c'est la raison pour laquelle son marché de traduction reste robuste jusqu'à aujourd'hui.

Encore une fois, nous croyons que ce que le Canada a pu faire en matière de la profession de traduction, le Nigéria doit être capable de le faire aussi. Mais pour que ceci ne soit pas un simple souhait, il faudrait que les parties prenantes dans l'industrie de traduction au Nigéria (le gouvernement, les traductologues, les traducteurs, les donneurs d'ouvrages, etc.) soient prêtes à travailler en concertation vers l'optimisation de la formation en traduction professionnelle et son institutionnalisation dans le système d'éducation du pays. Pour ce faire, les démarches et les stratégies à considérer constitueront nos pistes de réflexion dans une recherche ultérieure. 


\section{BIBLIOGRAPHIE}

AIRE, Victor. Les études franco-africaines au Canada: un témoignage personnalisé. Article non publié présenté lors du $15^{\mathrm{e}}$ congrès annuel de l'Association nigériane des enseignants universitaires de français (ANEUF), tenu à Ile-Ife du 9 au 14 mars 2014.

AMOSUN, Tundonu. Training and Management of Translators and Interpreters. EUREKA, vol. 3, no. 1, janvier, 2010, p. 69-80.

BRUNETTE, Louise. Marché de la traduction et universités : des évolutions nécessaires parallèles. Montréal, Canada : Circuit, no. 57, automne 1997. p. 11-12.

CLOUTIER, Pierre. Le marché de traduction. Montréal, Canada : Circuit, no. 118, hiver 2013, p. 9.

COHEN, Betty. Péril en la demeure. Montréal, Canada : Circuit, no. 118, hiver 2013.

Tarif au mot ou à l'heure, le débat est peut-être dépassé. Montréal, Canada : Circuit, no. 105, automne 2009, p. 10-11.

Le Québec, terre de traduction. 2013.

http://www.tradulex.com/articles/Cohen.pdf

86 DELISLE, Jean. La traduction raisonnée : manuel d'initiation à la traduction professionnelle, anglais, français : méthode par objectifs d'apprentissage, Ottawa : Presses de l'Université d'Ottawa, 2013.

DE VOS, Anne Marie. La tarification chez les traducteurs, terminologues et interprètes. Montréal, Canada : Circuit, no. 105, automne 2009, p. 6-7.

GAUTHIER, François. Un sondage révélateur. Montréal, Canada : Circuit, no. 105, automne 2009 , p. 12.

Immigration et communautés culturelles Québec. Exercer la profession de traducteur agréé, de terminologue agréé ou d'interprète agréé. www.immigration-quebec.gouv.qc.ca. Consulté le 2 mai, 2015.

JOHNSON, Stella. Prof. Ekundayo Simpson: the translator's advocate. Tunde AJIBOYE (éd.) Fore-runners of French in Nigeria, Ilorin: Info-links Publishers, 2014, p 273-284.

KELM, Gregory. Entrevue sur le marché de traduction au Québec. Accordée le jeudi 29 avril 2015.

LAVALLÉE, François. Salariat ou pratique privée. Montréal, Canada : Circuit, no. 92, été 2006, p. 5-6.

9.

. If it ain't broke, don't fix it. Montréal, Canada : Circuit, no. 105, automne 2009, p.

MESA, Anne-Marie. L'inspection professionnelle, ça ne fait pas mal!. Montréal, Canada : Circuit, no. 118, hiver 2013, p. 6-7. 
Nigeria Institute of Translators and Interpreters (NITI). http://www.nitinigéria.net. Consulté le 5 mai, 2015.

SÉGARD, Virginie. Le traducteur professionnel : une espace menacée ou la robotisation du monde. 2009. www.nikibar.com/publications/virgine-segard-le-traducteur-professionnel.html. Consulté le 3 mai, 2015.

SERVICE CANADA. Traducteurs, terminologues et interprètes. 2015. http://www.servicecanada.gc.ca/fra/qc/emploi_avenir/statistiques/5125.shtml. Consulté le 4 mai, 2015.

SIMPSON, Ekundayo. Manpower Needs and the Training of Translators in West Africa. Translation : Principles and Applications. Lagos/Abuja, Interlingual, 2007, p. 252-269.

VAILLANCOURT, François; TOUCHETTE, Christine: Le statut du français sur le marché du travail au Québec, de 1970 à 1993 : les revenus du travail, Toronto, Institut C.D. Howe, 2001.

VALENTINE, Egan; GUÉVEL, Zélie. Enseigner à traduire : un processus complexe. Montréal, Canada : Circuit, no.57, automne 1997, p. 10-11.

i Olusegun Afolabi holds a BA (French language) and MA (Translation) respectively from Obafemi Awolowo University and University of Lagos both in Nigeria. He is currently a Ph.D (Translation studies) candidate at the Université Laval, Québec city, Canada. His research interests include translators'/interpreters' training, history of translation, theory of translation and audio-visual translation. He also practices as a freelance translator and conference interpreter in Canada, having practiced same in his native country (Nigeria) for close to two decades. Curriculum Vitae. http://ulaval.academia.edu/SegunAfolabi/CurriculumVitae

RECEBIDO EM: 24 de janeiro de 2016

ACEITO EM: 13 de julho de 2016

PUBLICADO EM: Dezembro de 2016 